\title{
Inteligencias múltiples y rendimiento académico del área de matemáticas en estudiantes de educación básica primaria*
}

\author{
Multiple Intelligences and Academic Performance of Mathematics Area \\ in Elementary School Students
}

\author{
Raúl Prada Núñez¹, Gerson Adriano Rincón Álvarez², César Augusto Hernández Suárez³
}

Para citar este artículo: Prada, R., Rincón, G. A., Hernández, C. A. (2018). Inteligencias múltiples y rendimiento académico del área de matemáticas en estudiantes de educación básica primaria. Infancias Imágenes, 17(2), 163-175

\section{Resumen}

El objetivo de este trabajo fue determinar el grado de asociación existente entre los tipos de inteligencias y el rendimiento académico de los estudiantes en el área de matemáticas. Para ello, se conformó una muestra intencional de 539 estudiantes de educación básica primaria de cuatro instituciones educativas públicas de la ciudad de Cúcuta, Colombia. El instrumento elegido fue el cuestionario de detección de inteligencias múltiples de McKenzie (1999), el cual fue aplicado colectivamente en el aula. Además, se obtuvieron las calificaciones de los tres primeros periodos académicos en el área de matemáticas de los mismos estudiantes. Estos datos se sometieron a análisis estadístico descriptivo univariante y de correlaciones con estadísticos no paramétricos. Los resultados obtenidos destacan la relación significativa existente entre el grado de inteligencia lógico-matemática y el rendimiento académico en matemáticas, lo que corrobora los hallazgos de estudios latinoamericanos previos y los fundamentos del modelo de Gardner.

Palabras clave: inteligencia, lógica matemática, rendimiento escolar, educación básica, estudiante.
Recibido: 17-octubre-2017 / Aprobado: 21-marzo-2018

\begin{abstract}
The objective of this study was to determine the degree of association between the types of intelligences and the academic performance of the students in the area of mathematics. To this end, an intentional sample of 539 students of primary basic education of four public educational institutions of the city of Cúcuta, Colombia was formed. The instrument chosen was McKenzie's multiple intelligences detection questionnaire, which was applied collectively in the classroom. In addition, scores were obtained from the first three academic periods in the mathematics area of the students themselves. These data were submitted to univariate descriptive statistical analysis and correlations with non-parametric statisticians. The results obtained highlight the significant relationship between the degree of logical-mathematical intelligence and academic performance in mathematics, which corroborates the findings of previous Latin American studies and the fundamentals of the model of Gardner.
\end{abstract}

Keywords: intelligence, mathematical logic, academic performance, elementary school, student.

\footnotetext{
* Artículo de investigación derivado del proyecto "Influencia de las inteligencias múltiples en el rendimiento académico del área de matemáticas en estudiantes de educación básica primaria", contrato FINU-UFPS 034-2016 del Grupo de Investigación en Pedagogía y Prácticas Pedagógicas (GIPEPP) de la Universidad Francisco de Paula Santander (UFPS), finalizado en diciembre de 2016.1 Máster en Estadística y Optimización y Magister en Matemáticas con mención en Educación. Docente, Facultad de Educación, Artes y Humanidades, UFPS. Correo electrónico: raulprada@ufps.edu.co

2 Máster en Educación y Neuropsicología. Docente, Facultad de Educación, Artes y Humanidades, UFPS.

Correo electrónico: gersonadrianora@ufps.edu.co

Magíster en Enseñanza de las Ciencias con mención en Matemáticas. Docente, Facultad de Educación, Artes y Humanidades, UFPS.

Correo electrónico: cesaraugusto@ufps.edu.co
} 


\section{Introducción}

Tradicionalmente se ha considerado que el rendimiento académico de los estudiantes depende en buena medida de su inteligencia (Edel Navarro, 2003). Sin embargo, con frecuencia se encuentran estudiantes que incluso teniendo una buena capacidad intelectual y aptitudes adecuadas para el estudio no obtienen un rendimiento adecuado. También se da el caso de alumnos que tienen un mejor desempeño en un tipo de asignaturas que en otro (Jiménez, 2000).

Para la explicación de estos hechos se han propuesto múltiples teorías, una de las cuales ha resultado ser altamente esclarecedora: la de Gardner (1983) sobre inteligencias múltiples. Este autor sostiene que la inteligencia no es una capacidad única, sino que abarca una gran variedad de destrezas y habilidades que permiten enfrentar y resolver problemas de distinta naturaleza. De ahí que una persona puede resultar más hábil o eficaz para determinados asuntos que para otros. Por otra parte,

164 las distintas inteligencias poseen un importante carácter cultural, con lo cual cada sociedad estimula, deliberadamente o de manera inconsciente, el desarrollo de unas por encima de otras.

Como es obvio, las instituciones escolares constituyen el espacio que las sociedades han destinado para el desarrollo de la capacidad del alumno para enfrentar y resolver distintos problemas de modo que pueda adaptar su formación y comportamiento a las del resto de las personas con las que convive. El modo mediante el cual se evalúa si las metas parciales de la educación de un estudiante se van cumpliendo es su rendimiento académico, que además se emplea como parámetro para establecer la calidad de la educación de una institución, región o país. En este sentido, existen pruebas estandarizadas a nivel internacional que permiten a los gobiernos clarificar el nivel de la calidad educativa que se imparte y, si es el caso, establecer los correctivos necesarios para aumentarla.

Una de las pruebas más reconocidas es la diseñada por el Programa Internacional de Evaluación de Estudiantes (Pisa, por sus siglas en inglés), de la Organización para la Cooperación y el Desarrollo Económico (OCDE), que se realiza cada tres años e incluye las áreas de Matemática, Ciencias y
Lectura. De acuerdo con los resultados que periódicamente se publican, los países latinoamericanos ocupan posiciones muy bajas. Por ejemplo, en el 2012 la prueba se aplicó en 65 países. Chile, México, Uruguay, Costa Rica, Brasil, Argentina, Colombia y Perú ocuparon los puestos 51, 53, 55, 56, 58, 59, 62 y 65 respectivamente (Bos, Ganimian y Vegas, 2013). El puntaje promedio general de la prueba fue de 494 en Matemáticas, 496 en Lectura y 501 en Ciencias. Colombia obtuvo promedios de 376, 403 y 399 que, como es evidente, se encuentran muy por debajo del promedio general (percentil $10^{\circ}$ ).

La prueba también distribuye a los estudiantes de acuerdo con sus niveles de competencias. Los niveles 5 y 6 agrupan a los alumnos que demuestran un desempeño superior. En el extremo opuesto, el nivel 2 incluye a los alumnos con un nivel de competencia básico. Durante el año 2012 la prueba fue presentada por 9073 estudiantes colombianos distribuidos en 352 instituciones educativas oficiales y privadas y cuya edad para el momento era de 15 años. Dentro de este conglomerado, solo el $0.3 \%$ alcanzó los niveles 5 o 6 en el área de Matemáticas, mientras que en niveles inferiores a 2 se ubicó el $73.8 \%$ de quienes participaron en la prueba. Esto quiere decir que 74 de cada 100 estudiantes no lograron interpretaciones literales de los resultados de problemas matemáticos, ni demostraron la capacidad de utilizar fórmulas, algoritmos o procesos para resolver problemas de números enteros; tampoco reconocieron o interpretaron situaciones contextualizadas.

En este panorama de resultados no deseados en el entorno de colombiano, el departamento de Norte de Santander no constituye, ni mucho menos, una excepción. Un indicador de esta situación son los resultados de las pruebas Saber $3^{\circ}$ del 2014, según los cuales el $40 \%$ de las instituciones educativas de la ciudad de Cúcuta se ubicaron en las categorías de insuficiente y mínimo. A pesar de que las cifras no son buenas, los resultados están por encima de la media nacional, que fue un $20 \%$ insuficiente y 29\% mínimo (Icfes, 2015).

Los resultados contenidos en informes internacionales, nacionales y regionales ponen de manifiesto un problema de fondo que exige a los 
educadores respuestas urgentes pero al mismo tiempo profundamente razonadas. De ahí la necesidad de emprender investigaciones destinadas a establecer los factores que influyen en el rendimiento académico de los estudiantes colombianos con el fin de plantear, diseñar y evaluar programas de intervención basados en evidencia científica que den respuesta al problema del bajo aprovechamiento académico estudiantil en el área de Matemáticas.

Las dificultades para adquirir y demostrar habilidad matemática son, como cabe pensar tratándose de un tipo de pensamiento complejo, múltiples. Una seguramente tiene que ver con que los estudiantes han sido sometidos a lo largo de su proceso de escolarización a un sistema tradicional de enseñanza que ha favorecido la consideración de la Matemática como una asignatura de procesos repetitivos que no precisa de habilidades de razonamiento (Chaves, Castillo y Gamboa, 2008). Otra de las causas fundamentales del bajo rendimiento está asociada a la propia complejidad del objeto de estudio (Godino, 2002). Desde luego, estas no son las dos únicas causas de dificultad para el aprendizaje de las matemáticas y el bajo rendimiento académico de quienes las estudian. Lo que sí es cierto es que conviene reconocer y describir adecuadamente todos y cada uno de los factores que pueden estar incidiendo en la existencia de tales problemas.

En este sentido, esta investigación se ha propuesto establecer si existe relación entre el rendimiento matemático y los distintos tipos de inteligencia descritos por Gardner (1983). A fin de que fuera abarcable, la investigación se circunscribió a estudiantes de quinto grado de educación básica primaria de cuatro instituciones educativas de la ciudadela de Juan Atalaya en Cúcuta, Norte de Santander (Colombia). Concretamente, con la realización de la investigación se propuso responder la siguiente interrogante: ¿existe relación entre las inteligencias múltiples y el rendimiento académico en matemáticas de los estudiantes de quinto grado de educación básica primaria en Colombia?

El tema de las inteligencias múltiples ha despertado gran interés entre psicólogos y educadores, desde que fuera propuesto hasta nuestros días. Debido a la abundancia de materiales y documentos que se encuentran sobre esta temática es preciso establecer algunas delimitaciones teóricas sobre la concepción de inteligencia y de inteligencias múltiples, lo mismo que sobre la utilidad de estas definiciones para el trabajo de aula.

\section{Inteligencias múltiples}

Gardner (1983) propone una teoría muy interesante para clasificar la gran variedad de destrezas y habilidades presente en los seres humanos para solucionar problemas o crear instrumentos que faciliten la solución de dichos problemas en una o varias comunidades. Estas destrezas y habilidades son inteligencias diferenciadas (de ahí la denominación de múltiples) y relativamente independientes, por lo que un individuo puede desarrollar algunas más que otras. En todo caso, su reconocimiento permitirá idear y poner en práctica estrategias pedagógicas para su desarrollo.

Gardner (1983) considera que la inteligencia es la capacidad de la mente para construir productos que resuelvan problemas dentro de una o varias culturas. Lo anterior supone que la inteligencia no es un atributo completamente innato en el ser humano, sino que buena parte de lo que llamamos inteligencia consiste en un conjunto de capacidades, destrezas y habilidades que se desarrollan y reproducen dependiendo del contexto en el cual se vive.

La inteligencia es, pues, un fenómeno complejo en el que se interrelacionan la observación, la atención, la percepción, la memoria, el razonamiento, la imaginación, los deseos, las costumbres, la experiencia y la interacción social. Tales destrezas constituyen la base y fortalecen las inteligencias múltiples (Gardner, 1993).

Gardner (1993) enumera ocho inteligencias múltiples. Veamos cada una por separado:

\section{Inteligencia lingüística}

Destreza para utilizar la palabra y el lenguaje oral o por escrito de una manera altamente efectiva. Esta inteligencia concierne a la habilidad para elaborar y comprender mensajes mediante estructuras sintácticas y semánticas complejas y a través de un repertorio léxico rico y expresivo. Incluye, además, destreza retórica, mnemotécnica y metalingüística (uso del lenguaje para analizar el 
propio lenguaje). Contiene asimismo la capacidad de emplear el lenguaje con propósitos pragmáticos amplios y diversos. Este tipo de inteligencia se encuentra especialmente desarrollada en escritores, poetas, periodistas y oradores en general (maestros y profesores, predicadores, actores profesionales, etc.). Las personas muy lingüísticas piensan en palabras; les gusta leer, escribir, explicar historias, los juegos de palabras; necesitan libros, objetos para escribir, papel, periódicos, diálogo, conversación, debates e historias (Armstrong, 2006).

\section{Inteligencia lógico-matemática}

Esta inteligencia la presentan las personas con destreza para utilizar efectivamente los números y habilidad para llevar todo tipo de cuentas de manera reflexiva. Incluye la pasión por los razonamientos en las relaciones lógicas de conjunciones, disyunciones, implicación y equivalencia o los esquemas que estas proporcionan, abstracciones en funciones y facilidad para la comprensión de símbolos matemáticos. En otras palabras, se trata de personas que tienen destreza para establecer secuencias, razonamientos lógicos, realizar operaciones de conjuntos y agrupamientos, desenvoltura para la comprensión de teorías matemáticas, realizar progresiones y secuencias. Esta inteligencia se halla predominante en matemáticos, científicos, ingenieros y programadores informáticos, contadores y similares. Las personas muy lógico-matemáticas piensan razonando; les gusta experimentar, preguntar, resolver enigmas lógicos, calcular; necesitan materiales para experimentar, materiales científicos y para manipular, visitas a centros en los que se expliquen fenómenos científicos (como planetarios o museos de ciencias) (Armstrong, 2006).

\section{Inteligencia espacial}

Destreza para observar y comprender el mundo y los distintos ambientes de manera exacta, con facilidad para capacidad de pensar en tres dimensiones, con conciencia de percibir imágenes externas e internas, recrearlas, transformarlas o modificarlas, con sensibilidad por formas, líneas, colores, espacios y relaciones existentes entre todos estos elementos. Quienes poseen esta inteligencia poseen capacidad de orientarse de manera adecuada en un mapa y recorrerlo tanto mental como físicamente, es decir, decodificar la información gráfica y la trasladan a la realidad y viceversa. Las personas muy espaciales piensan en imágenes; les gusta diseñar, dibujar, visualizar, garabatear; necesitan arte, piezas de construcción, videos, películas, diapositivas, juegos de imaginación, laberintos, rompecabezas, libros ilustrados, visitas a museos de arte. Esta inteligencia es propia de arquitectos, ingenieros, diseñadores, geógrafos, pilotos y urbanistas (Armstrong, 2006).

\section{Inteligencia corporal-cinestésica}

También recibe el nombre de kinestésica. Quienes la poseen son personas con habilidades para utilizar con destreza el cuerpo y manipular objetos, lo cual significa que cuentan con una gran coherencia entre flexibilidad, destreza, equilibrio, velocidad y agilidad para solucionar problemas o expresar sentimiento e ideas por intermedio de su cuerpo o transformando cosas con sus manos. Está presente en los deportistas que manipulan objetos con sus manos o piernas, por ejemplo, futbolistas, basquetbolistas o esgrimistas o practicantes de artes marciales. También es propia de bailarines y acróbatas. Las personas muy cinéticas-corporales piensan a través de sensaciones corporales; les gusta bailar, correr, saltar, construir, tocar, gesticular; necesitan juegos de rol, teatro, movimiento, juegos de construcción, deporte y juegos físicos, experiencias táctiles y del aprendizaje manual (Armstrong, 2006).

\section{Inteligencia musical}

Capacidad de registrar, recrear e imitar música, por intermedio de la voz o un instrumento. A quienes poseen esta inteligencia se le facilita reproducir o crear tonos, ritmos y melodías tanto mental como físicamente, de tal forma que la acción de componer surge de forma natural. Las personas muy musicales piensan a través de ritmos y melodías; les gusta cantar, silbar, canturrear, crear ritmos con los pies y las manos, escuchar; necesitan cantar acompañados, asistir a conciertos, tocar algún instrumento en casa y en el colegio. Incluso en el caso de que no hayan sido educadas en la ejecución de un instrumento o la lectura de partituras, poseen la capacidad intuitiva para comprender el lenguaje musical (Armstrong, 2006). 


\section{Inteligencia interpersonal}

Capacidad de empatizar, de comprender a las otras personas, sus estados de ánimo, motivaciones, sentimientos e intenciones. Esta inteligencia incluye la habilidad para entender expresiones faciales, tonos de voz, posturas y gestos, y de responder de forma efectiva a estas señales en el momento preciso y justo. Esta inteligencia se encuentra en actores, políticos, vendedores exitosos y buenos docentes. Las personas muy interpersonales piensan transmitiendo ideas a otras personas; les gusta liderar, organizar, relacionarse, manipular, mediar, asistir a fiestas; necesitan amigos, juegos en grupo, reuniones sociales, actos colectivos, clubes y relaciones del tipo mentores/discípulos (Armstrong, 2006).

\section{Inteligencia intrapersonal}

Quienes poseen esta inteligencia son personas con capacidad de entendimiento y comprensión de sí mismos, de dirigir y organizar su vida sobre la base de la autocomprensión, autodisciplina y autoestima. Son personas que conocen sus alcances y limitaciones, reconocen sus emociones y las controlan y adaptan para guiar su conducta, identificando sus fortalezas y debilidades. Debido a lo anterior, son buenos psicólogos, líderes religiosos o filósofos. Las personas muy intrapersonales piensan en relación con sus necesidades, sentimientos y objetivos; les gusta establecer metas, mediar, soñar, planificar, reflexionar; necesitan lugares secretos, soledad, tener proyectos propios y tomar decisiones de manera reflexiva (Armstrong, 2006).

\section{Inteligencia naturalista}

Quienes poseen esta inteligencia son personas con gran sentimiento y amor por la naturaleza; que entienden y protegen el mundo natural, las plantas y los animales. Poseen la capacidad de reconocer, distinguir y relacionar ciertas especies como integrantes de un grupo, se le facilita y disfrutan de clasificar y reconocer las especies de animales y plantas. Por ello, suelen ser personas hábiles en el trabajo de cuidado de plantas y animales. En esta inteligencia se destacan los veterinarios, biólogos, expedicionarios, zoólogos y botánicos. Las personas muy naturalistas piensan a través de la naturaleza y las formas naturales; les gusta jugar con sus mascotas, la jardinería, investigar la naturaleza, criar animales, cuidar del planeta. Necesitan tener acceso a la naturaleza, oportunidades para relacionarse con animales y herramientas para investigar la naturaleza (Armstrong, 2006).

Definidos los distintos tipos de inteligencia, es necesario ahora establecer su vínculo con el rendimiento académico.

\section{Rendimiento académico}

El rendimiento académico se define como el nivel del logro alcanzado por un estudiante en un medio ambiente educativo en general, o asignatura en particular, y se mide por intermedio de evaluaciones pedagógicas, enmarcadas en conjuntos de procedimientos planificados y aplicados dentro del proceso educativo. Por lo tanto, el fin de la evaluación consiste primordialmente en valorar los alcances del estudiante con respecto a los objetivos establecidos por dicho proceso (Vega, 1998, citado por Fuentes et al., 2015).

El propósito de la medición del rendimiento académico consiste en establecer si se ha alcanzado un objetivo de formación, una meta educativa, un aprendizaje. El aprendizaje que se promueve en la escuela implica la trasformación de un estado determinado de conocimiento del estudiante en un estado nuevo. Este cambio de estructuras cognitivas se alcanza mediante la integración de procesos mentales diversos y complejos. El rendimiento varía de acuerdo con las circunstancias, condiciones orgánicas y ambientales que determinan las aptitudes y experiencias. Cuando se produce un desfase entre el rendimiento académico y el rendimiento que se espera del alumno, se habla de rendimiento discrepante o insatisfactorio, como aquel que se sitúa por debajo del rendimiento esperado (Lamas, 2015).

La medición y mejoramiento del rendimiento académico implica procesos complejos, por lo que su abordaje debe hacerse necesariamente a partir de su consideración como fenómeno multifactorial que incluye aspectos tales como el nivel intelectual, la personalidad, la motivación, las aptitudes, los intereses, los hábitos de estudio, la autoestima y la relación profesor-alumno (Lamas, 2015), los factores socioeconómicos, afectivo, motivacionales, emocionales, influidos por las expectativas de 
los familiares, docentes y los mismos estudiantes frente a los logros deseados en el aprendizaje; los objetivos y perfiles de los planes y programas de estudio, las metodologías, pedagogías y didácticas empleadas en la enseñanza, los preconceptos o conocimientos previos que tienen los estudiantes (Benítez, Giménez y Osicka, 2000).

\section{Relación entre inteligencias múltiples y rendimiento académico}

Por mucho tiempo se había pensado que la inteligencia medida en términos de cociente intelectual y la voluntad eran los únicos factores asociados al rendimiento académico, pero en diversas investigaciones se ha demostrado que esta relación solo explica entre el $10 \%$ y $20 \%$ del éxito académico. De lo anterior se deduce que un alto porcentaje (entre el $80 \%$ y el $90 \%$ ) del rendimiento debe ser explicado a través de factores, entre los que posiblemente se encuentra la inteligencia emocional como parte de las inteligencias múltiples (Otero, Martín, León del Barco y Vicente, 2009).

Respecto de la relación entre inteligencia y rendimiento académico, se puede afirmar que:

La inteligencia humana no es una realidad fácilmente identificable, es un constructo utilizado para estimar, explicar o evaluar algunas diferencias conductuales entre las personas: éxitos/fracasos académicos, modos de relacionarse con los demás, proyecciones de proyectos de vida, desarrollo de talentos, notas educativas, resultados de test cognitivos, etc. (Crespo y Pizarro, 1998, p. 14)

El rendimiento o desempeño escolar está efectivamente asociado a la inteligencia, pero también queda claro que existen múltiples factores que le afectan, positiva y negativamente (Fuentes et al., 2015).

Revisemos algunas de las investigaciones que han mostrado esta relación:

Rincón (2005), en su proyecto inteligencias múltiples y rendimiento académico en alumnos de educación básica, halló correlación positiva significativa entre inteligencias múltiples y rendimiento académico; de su investigación sobresale que la inteligencia matemática era predictora de buen rendimiento académico.
Otros estudios han confirmado la correlación positiva y significativa de la inteligencia lógico-matemática con el desempeño académico general, pero especialmente con el rendimiento en matemáticas. En efecto, se evidencian niveles significativamente mayores en la inteligencia lógico-matemática de estudiantes talentosos (Andrade, Freixas y Miranda, 2001; Lynn y Mikk, 2009; Núñez y Lozano, 2005; Núñez et al., 2007; Ferrándiz, Bermejo, Sainz, Ferrando y Prieto, 2008; Kornilova, Kornilov y Chumakova, 2009; Stock, Desoete y Roeyers, 2009; Ferrándiz et al., 2010; Cerda, Ortega, Pérez, Flores y Melipillán, 2011).

García (2012) consiguió una correlación positiva significativa entre la inteligencia emocional intrapersonal y el rendimiento académico. Entre los resultados de la investigación se encuentra que los estudiantes con alto nivel de inteligencia emocional intrapersonal pertenecían al grupo de alto rendimiento académico, mientras que los que tenían bajo nivel de esta inteligencia tenían también bajo rendimiento académico.

Aquellas personas que son capaces de adaptarse y resolver problemas de una manera más eficiente y adecuada a una cultura determinada tienden a tener varias inteligencias predominantes (Gardner, 1999). De lo anterior se puede inferir que estudiantes con una mayor cantidad de inteligencias predominantes alcanzarán un alto rendimiento académico.

Diferentes investigaciones han hallado relaciones entre inteligencia matemática y rendimiento académico en matemáticas. Así, por ejemplo, Fernández, García y González (2013) hallaron que los sujetos con mejor rendimiento en matemáticas presentaban un mayor conocimiento de las estrategias de autorregulación como la planificación, ejecución y evaluación, habilidades propias de la inteligencia matemática. Estos resultados se asemejan a los de Elosúa et al. (2012), quienes, en su estudio de una muestra de alumnos de tercero y sexto año de primaria, encontraron relación entre las habilidades lectoras de decodificación y las habilidades de comprensión y el rendimiento escolar en lengua y matemáticas.

Llor et al. (2012), en un estudio con alumnos de edades comprendidas entre 11 y 17 años, encontraron correlaciones positivas y estadísticamente significativas entre las inteligencias naturalista, 
lógico-matemática y lingüística y el rendimiento académico en las materias de Ciencias de la Naturaleza y Biología y Geología, Matemáticas y Lengua Castellana y Literatura. Sin embargo, otros estudios indican que los alumnos con alto rendimiento no presentan necesariamente niveles altos de las inteligencias mencionadas anteriormente. De hecho, los estudiantes superdotados presentan sus puntos fuertes en inteligencias interpersonal y musical (Chan, 2004).

\section{Metodología}

\section{Nivel y diseño de la investigación}

Esta es una investigación cuantitativa y el diseño utilizado es el de una investigación no experimental (ex-post-facto) de tipo correlacional (Carrasco y Caldero, 2000). Las variables que se correlacionan en este estudio son los ocho tipos de inteligencias múltiples y rendimiento académico en el área de Matemáticas. Dado que las variables del estudio se examinaron en categorías, el análisis de las correlaciones se basó en la aplicación del coeficiente de correlación Tau-c de Kendall como medida no paramétrica de asociación para variables ordinales que ignora los empates.

La hipótesis de trabajo (o nula) que se sometió a validación durante el estudio se basó en probar la existencia de alguna relación entre las principales variables del estudio, esto es:

- Ho: los distintos tipos de inteligencias múltiples no se relacionan con el rendimiento académico del estudiante en el área de Matemáticas.

- Ha: al menos un tipo de inteligencia está relacionado con el rendimiento académico del estudiante en el área de Matemáticas.

\section{Población y muestra}

La población objeto de estudio estuvo conformada por los estudiantes colombianos entre 10 y 13 años que durante el año 2016 se encontraban matriculados en el quinto grado de educación básica primaria, en las diversas instituciones educativas públicas y privadas de Cúcuta, Norte de Santander, Colombia.
La selección se realizó utilizando el muestreo no probabilístico bajo la técnica de muestreo intencional. La selección de la técnica es apropiada para el estudio puesto que se propuso establecer la eventual relación entre el rendimiento académico y las diversas inteligencias múltiples. En este sentido, se seleccionaron cuatro instituciones públicas de la ciudadela de Juan Atalaya. Se decidió trabajar con estudiantes de escasos recursos debido a que el propósito general de la investigación fue indagar la presencia innata de las inteligencias múltiples en entornos poco motivantes. Como criterio de exclusión se consideró el padecimiento de alguna necesidad educativa especial o estar sometido a adaptaciones curriculares.

Finalmente, la muestra quedó compuesta por 539 estudiantes (277 de género femenino y 262 masculino) con edades comprendidas entre 10 y 13 años (media = 11.19; desviación estándar = 1.38).

\section{Instrumentos de recolección de información}

Para la recolección de información y medición de las inteligencias múltiples se utilizó la adaptación y contextualización del test de detección de las inteligencias múltiples de Mackenzie (1999), en su traducción española. El cuestionario evalúa las ocho inteligencias propuestas por Gardner (1983). Cada inteligencia se evalúa a través de 10 ítems, con una escala Likert de 3 puntos ( $1=$ de acuerdo; $0.5=$ a veces; 0 = desacuerdo). El protocolo de interpretación indica que se deben sumar los puntos obtenidos en cada uno de los ítems, con lo cual se establece una puntuación por cada inteligencia. En función de los resultados de las puntuaciones se obtienen los niveles que poseen los estudiantes en las distintas inteligencias. Esta escala cuantitativa puede ser transformada a otra cualitativa (tabla 1):

Tabla 1. Niveles de valor para los puntajes de las inteligencias múltiples

\begin{tabular}{|c|c|}
\hline Puntaciones & Niveles \\
\hline 0 a 2 & Bajo \\
\hline 2,5 a 4 & Medio-bajo \\
\hline 4.5 a 6 & Medio \\
\hline 6.5 a 8 & Medio-alto \\
\hline 8.5 a 10 & Alto \\
\hline
\end{tabular}

Fuente: Mackenzie (1999). 
La adaptación de McKenzie (1999) del cuestionario de detección de las inteligencias múltiples ha sido empleada por numerosos autores tales como Galera (2015), debido a que el test constituye una forma muy cómoda y fiable de calcular el nivel de cada inteligencia. Por otra parte, la validez del test ha sido comprobada por numerosos autores, entre los cuales cabe citar a Hajhashemi y Eng (2010) o Prieto y Ballester (2003). No obstante, para garantizar la validez del cuestionario en el presente estudio se volvió a calcular el índice de confiabilidad mediante la aplicación del alfa de Cronbach para la escala global. El valor obtenido es de $\alpha=0.90$, resultado que se traduce en un nivel muy alto de confiabilidad.

En relación con la medición del rendimiento académico, se procedió inicialmente a la recolección de datos de calificaciones de los estudiantes en el área de Matemáticas, utilizando como fuente de información secundaria los registros que reposan en la Secretaría Académica de cada institución educativa

170 y que resumen el rendimiento de los estudiantes durante los tres primeros períodos del año lectivo 2016.

\section{Procedimiento}

Se comentó al rector de cada institución educativa el objetivo del proyecto. En las instituciones cuyos directivos manifestaron el deseo de colaboración se realizó una reunión con estudiantes y sus padres de familia con el fin de explicar la actividad que se desarrollaría luego y obtener de este modo el consentimiento por escrito y el deseo de formar parte del estudio. Las pruebas de inteligencias múltiples se realizaron con el apoyo de los docentes titulares de cada grupo en la jornada de la mañana. La actividad inició en cada escuela a las 6:30 am y concluyó a las 11:00 am.

\section{Procedimientos de análisis}

La información obtenida mediante la aplicación del test se procesó de forma cuantitativa. En un inicio se realizó un informe descriptivo por medio de tablas cruzadas en el que se muestran las tendencias de los datos. Posteriormente se realizó un análisis de correlaciones entre las variables categóricas considerando dos niveles (alto y bajo) para la variable rendimiento académico y los distintos tipos de inteligencias múltiples. Los análisis estadísticos se realizaron con el software SPSS versión 23.0.

El signo del coeficiente establece la dirección de la relación y su valor absoluto indica la fuerza de la relación. Los valores mayores señalan que la relación es más estrecha. Los valores posibles van de $-1 \mathrm{a}+1$, pero un valor de $-1 \mathrm{o}+1$ solo se puede obtener a partir de tablas cuadradas. Cuanto más próximos estén los valores de estas medidas a 0 , más débil será la asociación entre las variables. Cuanto más cercanos a 1 (o a -1) sean los valores de todas estas medidas, mayor será la asociación positiva (o negativa) entre las variables.

\section{Resultados}

Los resultados muestran en principio un análisis descriptivo del conjunto de datos para cada variable de interés realizado en función de los puntajes originales. Luego, se evalúan las correlaciones utilizando las categorías asociadas a cada puntuación tal y como se explicó en el apartado metodológico.

La tabla 2 muestra los estadísticos descriptivos correspondientes a las puntuaciones totales obtenidas por los estudiantes en cada tipo de inteligencia. En general, los valores medios son muy similares y se ubican en el rango de medio alto a alto. Los valores medios más elevados corresponden a las inteligencias naturalista, lógico-matemática e intrapersonal, con 7.8 puntos. La puntuación más baja recae sobre la inteligencia musical, con un valor de 7.6.

En relación con el rendimiento académico, su valor promedio fue de 3.4 puntos, con una baja variabilidad equivalente a 0.6 puntos. Los datos fueron analizados en función de dos grupos definidos por

Tabla 2. Estadísticos descriptivos para

los distintos tipos de inteligencia

\begin{tabular}{|l|c|c|c|c|c|}
\hline & Media & Med & DT & Mín & Máx \\
\hline Naturalista & 7.8 & 8.0 & 1.6 & 1.0 & 10.0 \\
\hline Musical & 6.7 & 7.0 & 1.7 & 2.0 & 10.0 \\
\hline Lógico-matemática & 7.8 & 8.0 & 1.4 & 1.0 & 10.0 \\
\hline Interpersonal & 7.7 & 8.0 & 1.5 & 2.0 & 10.0 \\
\hline Cinestésica & 7.5 & 7.5 & 1.6 & 1.0 & 10.0 \\
\hline Lingüística & 7.6 & 8.0 & 1.7 & .0 & 10.0 \\
\hline Intrapersonal & 7.8 & 8.0 & 1.5 & .0 & 10.0 \\
\hline Espacial & 7.6 & 7.5 & 1.5 & .0 & 10.0 \\
\hline $\begin{array}{l}\text { Rendimiento en Mate- } \\
\text { máticas }\end{array}$ & 3.4 & 3.4 & .6 & 1.0 & 5.0 \\
\hline
\end{tabular}

Fuente: elaboración propia de los autores. 
su rendimiento académico en el área de Matemáticas: en una escala de evaluación de 1 a 5, se asignó el valor de bajo para aquellos con menos de 3 y alto para valores por encima de 3. De acuerdo con este análisis, el $73.3 \%$ de los estudiantes que formaron parte de la investigación se agrupan en el nivel bajo de rendimiento académico en Matemáticas. La tabla 3 resume la relación entre los resultados de cada tipo de inteligencia con los niveles de rendimiento académico de los estudiantes en el área de Matemática.

Tabla 3. Distribución de frecuencias conjuntas entre los niveles por tipos de inteligencia y los niveles de rendimiento en el área de Matemáticas

\begin{tabular}{|c|c|c|c|c|c|c|c|}
\hline & & \multicolumn{6}{|c|}{ Nivel de rendimiento en Matemáticas } \\
\hline & \multicolumn{2}{|c|}{ Alto } & \multicolumn{2}{|c|}{ Bajo } & \multicolumn{2}{|c|}{ Total } & \\
\hline & $f$ & $\%$ & $f$ & $\%$ & f & $\%$ & \\
\hline \multirow{6}{*}{ Naturalista } & Bajo & 3 & $0.6 \%$ & 9 & $1.7 \%$ & 12 & $2.2 \%$ \\
\hline & Medio-bajo & 1 & $0.2 \%$ & 5 & $0.9 \%$ & 6 & $1.1 \%$ \\
\hline & Medio & 15 & $2.8 \%$ & 28 & $5.2 \%$ & 43 & $8.0 \%$ \\
\hline & Medio-alto & 67 & $12.4 \%$ & 158 & $29.3 \%$ & 225 & $41.7 \%$ \\
\hline & Alto & 58 & $10.8 \%$ & 195 & $36.2 \%$ & 253 & $46.9 \%$ \\
\hline & Total & 144 & $26.7 \%$ & 395 & $73.3 \%$ & 539 & $100.0 \%$ \\
\hline \multirow{6}{*}{ Musical } & Bajo & 1 & $0.2 \%$ & 1 & $0.2 \%$ & 2 & $0.4 \%$ \\
\hline & Medio-bajo & 11 & $2.0 \%$ & 37 & $6.9 \%$ & 48 & $8.9 \%$ \\
\hline & Medio & 43 & $8.0 \%$ & 104 & $19.3 \%$ & 147 & $27.3 \%$ \\
\hline & Medio-alto & 61 & $11.3 \%$ & 190 & $35.3 \%$ & 251 & $46.6 \%$ \\
\hline & Alto & 28 & $5.2 \%$ & 63 & $11.7 \%$ & 91 & $16.9 \%$ \\
\hline & Total & 144 & $26.7 \%$ & 395 & $73.3 \%$ & 539 & $100.0 \%$ \\
\hline \multirow{6}{*}{ Lógica-Matemática } & Bajo & 2 & $0.4 \%$ & 0 & $0.0 \%$ & 2 & $0.4 \%$ \\
\hline & Medio-bajo & 2 & $0.4 \%$ & 4 & $0.7 \%$ & 6 & $1.1 \%$ \\
\hline & Medio & 9 & $1.7 \%$ & 49 & $9.1 \%$ & 58 & $10.8 \%$ \\
\hline & Medio-alto & 60 & $11.1 \%$ & 192 & $35.6 \%$ & 252 & $46.8 \%$ \\
\hline & Alto & 71 & $13.2 \%$ & 150 & $27.8 \%$ & 221 & $41.0 \%$ \\
\hline & Total & 144 & $26.7 \%$ & 395 & $73.3 \%$ & 539 & $100.0 \%$ \\
\hline \multirow{6}{*}{ Interpersonal } & Bajo & 0 & $0.0 \%$ & 1 & $0.2 \%$ & 1 & $0.2 \%$ \\
\hline & medio-bajo & 5 & $0.9 \%$ & 8 & $1.5 \%$ & 13 & $2.4 \%$ \\
\hline & Medio & 18 & $3.3 \%$ & 51 & $9.5 \%$ & 69 & $12.8 \%$ \\
\hline & Medio-alto & 64 & $11.9 \%$ & 177 & $32.8 \%$ & 241 & $44.7 \%$ \\
\hline & Alto & 57 & $10.6 \%$ & 158 & $29.3 \%$ & 215 & $39.9 \%$ \\
\hline & Total & 144 & $26.7 \%$ & 395 & $73.3 \%$ & 539 & $100.0 \%$ \\
\hline \multirow{6}{*}{ Cinestésica } & Bajo & 1 & $0.2 \%$ & 5 & $0.9 \%$ & 6 & $1.1 \%$ \\
\hline & Medio-bajo & 4 & $0.7 \%$ & 7 & $1.3 \%$ & 11 & $2.0 \%$ \\
\hline & Medio & 35 & $6.5 \%$ & 62 & $11.5 \%$ & 97 & $18.0 \%$ \\
\hline & Medio-alto & 59 & $10.9 \%$ & 181 & $33.6 \%$ & 240 & $44.5 \%$ \\
\hline & Alto & 45 & $8.3 \%$ & 140 & $26.0 \%$ & 185 & $34.3 \%$ \\
\hline & Total & 144 & $26.7 \%$ & 395 & $73.3 \%$ & 539 & $100.0 \%$ \\
\hline \multirow{6}{*}{ Lingüística } & Bajo & 2 & $0.4 \%$ & 1 & $0.2 \%$ & 3 & $0.6 \%$ \\
\hline & Medio-bajo & 4 & $0.7 \%$ & 11 & $2.0 \%$ & 15 & $2.8 \%$ \\
\hline & Medio & 24 & $4.5 \%$ & 67 & $12.4 \%$ & 91 & $16.9 \%$ \\
\hline & Medio-alto & 60 & $11.1 \%$ & 168 & $31.2 \%$ & 228 & $42.3 \%$ \\
\hline & Alto & 54 & $10.0 \%$ & 148 & $27.5 \%$ & 202 & $37.5 \%$ \\
\hline & Total & 144 & $26.7 \%$ & 395 & $73.3 \%$ & 539 & $100.0 \%$ \\
\hline \multirow{6}{*}{ Intrapersonal } & Bajo & 1 & $0.2 \%$ & 0 & $0.0 \%$ & 1 & $0.2 \%$ \\
\hline & Medio-bajo & 2 & $0.4 \%$ & 7 & $1.3 \%$ & 9 & $1.7 \%$ \\
\hline & Medio & 19 & $3.5 \%$ & 52 & $9.6 \%$ & 71 & $13.2 \%$ \\
\hline & Medio-alto & 69 & $12.8 \%$ & 181 & $33.6 \%$ & 250 & $46.4 \%$ \\
\hline & Alto & 53 & $9.8 \%$ & 155 & $28.8 \%$ & 208 & $38.6 \%$ \\
\hline & Total & 144 & $26.7 \%$ & 395 & $73.3 \%$ & 539 & $100.0 \%$ \\
\hline \multirow{6}{*}{ Espacial } & Bajo & 1 & $0.2 \%$ & 0 & $0.0 \%$ & 1 & $0.2 \%$ \\
\hline & Medio-bajo & 5 & $0.9 \%$ & 6 & $1.1 \%$ & 11 & $2.0 \%$ \\
\hline & Medio & 24 & $4.5 \%$ & 72 & $13.4 \%$ & 96 & $17.8 \%$ \\
\hline & Medio-alto & 76 & $14.1 \%$ & 178 & $33.0 \%$ & 254 & $47.1 \%$ \\
\hline & Alto & 38 & $7.1 \%$ & 139 & $25.8 \%$ & 177 & $32.8 \%$ \\
\hline & Total & 144 & $26.7 \%$ & 395 & $73.3 \%$ & 539 & $100.0 \%$ \\
\hline
\end{tabular}

Fuente: elaboración propia de los autores. 
La tabla 3 muestra una autopercepción alta en la mayoría de las inteligencias, destacando en el $88 \%$ de los casos de valoraciones de medio alto a alto con respecto a la inteligencia naturalista y lógico-matemática, seguida por una puntuación del 85\% para las inteligencias intrapersonal e interpersonal. En relación con el rendimiento académico, resalta una ponderación alta en las inteligencias naturalista y lógico-matemática, con aproximadamente un $24 \%$. Les siguen las inteligencias intra e interpersonal $(22 \%)$. Al evaluar la concentración de los casos con altos niveles de rendimiento en matemática aparecen con una representación significativa las inteligencias lingüística y espacial, con una representación cercana al $21 \%$ de los casos en cada renglón.

Con el fin de dar respuesta a la hipótesis principal de la investigación, se procedió a realizar el análisis bivariante entre cada uno de los niveles de las variables, tipo de inteligencia particular y nivel de rendimiento en matemática; evaluando de esta manera la posible relación de dependencia entre 172 los diversos niveles de cada uno de ellos. Los resultados aparecen en la tabla 4.

Tabla 4. Tau-c de Kendall entre nivel de rendimiento en matemáticas vs. niveles de inteligencia

\begin{tabular}{|l|c|c|}
\hline Inteligencia & Tau-c de Kendall & P-valor \\
\hline Naturalista & -0.076 & 0.054 \\
\hline Musical & 0.006 & 0.877 \\
\hline Lógico-matemática & 0.105 & 0.008 \\
\hline Interpersonal & -0.006 & 0.883 \\
\hline Cinestésica & -0.083 & 0.050 \\
\hline Lingüística & -0.006 & 0.890 \\
\hline Intrapersonal & -0.018 & 0.650 \\
\hline Espacial & -0.650 & 0.103 \\
\hline
\end{tabular}

Fuente: elaboración propia de los autores.

Puesto que se trata de variables ordinales de diferentes rangos, se aplica la prueba de hipótesis con el estadístico Tau-c de Kendall, el cual plantea como hipótesis nula la no existencia de asociación entre los niveles de los distintos tipos de inteligencia y los grupos de alto y bajo rendimiento académico en matemáticas:

- Al evaluar la inteligencia lógico-matemática se obtuvo un estadístico cuyo valor es de 0.105 y su p-valor de $0.008<0.05$, lo que conlleva a rechazar la hipótesis de trabajo de independencia de variables. Ello indica que hay evidencia estadística suficiente para rechazar la hipótesis nula y aceptar que existe relación entre los grupos de alto y bajo rendimiento académico en Matemáticas y este tipo de inteligencia.

- Para el resto de las inteligencias (naturalista, musical, interpersonal, cinestésica, lingüística, intrapersonal y espacial) el $p$-valor es $\geq 0.05$, con lo cual se acepta la hipótesis de que existe evidencia estadística suficiente para indicar que este tipo de inteligencias no están relacionadas con el rendimiento académico de los estudiantes en el área de matemáticas.

\section{Conclusiones}

Los resultados indican una correlación significativa entre la inteligencia lógica matemática y el rendimiento en esta asignatura. En este sentido, los hallazgos confirman una tendencia demostrada en otras investigaciones del contexto latinoamericano (Cerda et al., 2011; Aliaga et al., 2012). De este modo, queda claro que en la mayoría de los casos este tipo de inteligencia se asocia más con el rendimiento en Matemática que con el rendimiento general del alumno.

Desde los propios fundamentos de las inteligencias múltiples, Gardner (2001) señala que esta relación entre rendimiento en Matemática con la inteligencia lógico-matemática no sorprende en lo absoluto, e indica que la teoría de las inteligencias múltiples se concibió como una descripción de los individuos en función de su pasado evolutivo y su supervivencia en nichos ecológicos y culturales. Por lo tanto, es natural que la expresión de la inteligencia lógico-matemática sobresalga en la autopercepción del estudiante en relación con sus experiencias vividas.

Por otra parte, Guzmán y Castro (2005) señalan que todos los estudiantes poseen todos los tipos de inteligencia en sus diferentes grados de desarroIlo a nivel individual y colectivo. El desarrollo de un tipo o más tipos de inteligencia probablemente está condicionado por las preferencias y estas, a su vez, inciden en el aprendizaje. Entre los estudiantes participantes del estudio se evidencia un desarrollo más bien homogéneo de las inteligencias 
naturalista, lógico-matemática, interpersonal, intrapersonal y espacial. Con porcentajes superiores al $80 \%$, se ubican en un nivel promedio medio-alto y alto, lo cual indica que estos estudiantes poseen un importante potencial de competencias para el cuidado del medio ambiente, el razonamiento matemático, sensibilidad para relacionarse tanto con otras personas como consigo mismos y ubicación espacial. No obstante, Ilama la atención que la inteligencia musical resultara con los valores más bajos; estos alcanzan solamente a un nivel medio en relación con las puntuaciones generales.

La inteligencia lógico-matemática influye en la conformación de los grupos de alto y bajo rendimiento en matemáticas, lo que indica que existe una relación entre las puntuaciones de la inteligencia lógico-matemática y el rendimiento académico en matemáticas (Ramos-Amaya y Martínez-Berruezo, 2015; Morales, 2013; Llor et al., 2012); pero las inteligencias naturalista, musical, interpersonal, intrapersonal, lingüística, espacial y cinestésica no resultaron influyentes en la conformación de los grupos de alto y bajo rendimiento en matemáticas.

Las evaluaciones realizadas en el contexto del estudio reafirman la relación entre el nivel de inteligencia lógico-matemática y el rendimiento académico en esta área. Se destaca una relación significativa solo entre esta dupla y no entre el rendimiento en relación los otros tipos de inteligencia analizados. Por este motivo, se puede afirmar que en la medida en que los estudiantes muestren una puntuación más alta en su inteligencia lógico-matemática, en correspondencia, su nivel de rendimiento académico en esta asignatura será también alto.

\section{Referencias}

Andrade, M., Freixas, I. y Miranda, C. (2001). Predicción del rendimiento académico lingüístico y lógico matemático por medio de las variables modificables de las inteligencias múltiples del hogar. Boletín Investigación Educacional, 16, 301-315.

Aliaga, J., Ponce, C., Bulnes, M., Elizalde, R., Montgomery, W., Gutiérrez, V. y Torchiani, R. (2012). Las inteligencias múltiples: evaluación y relación con el rendimiento en matemática en estudiantes del quinto año de secundaria de
Lima Metropolitana. Revista de investigación en Psicología, 15(2), 163-202. DOl: https://doi. org/10.15381/rinvp.v15i2.3699

Armstrong, T. (2006). Inteligencias múltiples cómo descubrirlas y estimularlas en sus hijos. Bogotá: Norma.

Benítez, M., Giménez, M. y Osicka, R. (2000). Las asignaturas pendientes y el rendimiento académico: ¿existe alguna relación? Recuperado de http://www.unne.edu.ar/unnevieja/Web/cyt/ cyt/humanidades/h-009.pdf

Bos, M. S., Ganimian, A. J. y Vegas, E. (2013). Brief \#1: ¿Cómo le fue a la región? Serie de Briefs - América Latina en Pisa 2012. Washington; París: Inter-American Development Bank and Organization for Economic Cooperation and Development.

Carrasco, J. B. y Caldero, J. F. (2000). Aprendo a investigar en educación. Madrid: Rialp.

Cerda, G., Ortega, R., Pérez, C., Flores, C. y MelipiIlán, R. (2011). Inteligencia lógica y rendimiento académico en matemáticas: un estudio con estudiantes de educación básica y secundaria de chile. Anales de Psicología, 1, 389-398.

Chan, D. W. (2004). Multiple intelligences of Chinese gifted students in Hong Kong: perpectives from students, parents, teachers and peers. Roeper Review, 27, 1-18. DOI: https://doi. org/10.1080/02783190409554284

Chaves, E., Castillo, M. y Gamboa, R. (2008). Creencias de los estudiantes en los procesos de aprendizaje de las matemáticas. Cuadernos de Investigación y Formación en Educación Matemática, 3(4), 29-44.

Crespo, N. y Pizarro, R. (1998). Inteligencias múltiples y aprendizajes escolares. Investigación Educativa, 2(3), 69-74.

Edel Navarro, R. (2003). El rendimiento académico: concepto, investigación y desarrollo. Reice. Revista Iberoamericana sobre Calidad, Eficacia y Cambio en Educación, 1(2).

Elosúa, M. R., García, J. A., Gómez, 1., López, C., Pérez, E. y Orjales, I. (2012). Habilidades lectoras y rendimiento académico en 30 y 60 de primaria: aspectos evolutivos y educativos. Estudios de Psicología, 33(2), 207-218. DOI: https://doi.org/10.1174/021093912800676411 
Fernández, M. S., García, T. y González, P. (2013). Autorregulación y rendimiento académico en matemáticas. Aula Abierta, 41(1), 39-48.

Ferrándiz, C., Bermejo, R., Sainz, M., Ferrando, M. y Prieto, M. D. (2008). Estudio del razonamiento lógico-matemático desde el modelo de las inteligencias múltiples. Anales de Psicología, 24(2), 213-222.

Ferrándiz, C., Prieto, D., Fernández, C., Soto, G., Ferrando, M. y Badía, M. (2010). Modelo de identificación de alumnos con altas habilidades de educación secundaria. Revista Electrónica Interuniversitaria de Formación del Profesorado, 13(1), 63-74

Fuentes, S., Velázquez, M. J., Abarca, M. S., Covarrubias, M. L. y Figueroa, M. A. (2015). Análisis de la relación entre creatividad, inteligencia múltiple y rendimiento académico de estudiantes de un Bachillerato en Artes del Estado de Colima, México. Revista Internacional de Pedagogía y Currículo, 2(15), 14-25.

Galera, E. M. (2015). Relación entre inteligencias múltiples, creatividad y rendimiento académico en matemáticas para la elección de materias optativas. Badajoz, España: AnthropiQa 2.0 Lulu Press Inc.

García, C. (2012). Inteligencias múltiples, creatividad y rendimiento académico en educación secundaria (trabajo final de máster). Universidad Internacional de La Rioja, Badajoz, España.

Gardner, H. (1983). Estructuras de la mente. La teoría de las inteligencias múltiples. México: Fondo de Cultura Económica.

Gardner, H. (1993). Inteligencias múltiples. La teoría en la práctica. Barcelona: Paidós.

Gardner, H. (1999). Mentes extraordinarias. Barcelona: Kairós.

Gardner, H. (2001). La inteligencia reformulada: las inteligencias múltiples en el siglo XXI. Barcelona: Paidós.

Godino, J. D. (2002). Un enfoque ontológico y semiótico de la cognición matemática. Recherches en Didactique des Mathématiques, 22(2-3), 237-284.

Hajhashemi, K. y Eng, W. B. (2010). A validation study of the Persian version of McKenzie's multiple intelligences inventory to measure profiles of pre-university students. Pertanika Journal of Social Sciences \& Humanities (JSSH), 18(2), 343-355.

Icfes. (2015). Presentación uso de resultados prueba Saber 3, 5 y 9 Cúcuta 2015. Recuperado de http://www.icfes.gov.co/docman/talleres-y-jornadas-de-divulgacion/ saber-3-5-y-9-divulgaciones/divulgacion-2015/ taller-saber-3-5-9-2016/1467-presentacionanalisis-de-resultados-prueba-saber-3-5-y-9cucuta-2015/file?force-download=1

Jiménez, M. (2000). Competencia social: intervención preventiva en la escuela. Infancia y Sociedad, 24, 21-48.

Kornilova, T., Kornilov, S. y Chumakova, M. (2009). Subjective evaluations of intelligence and academic self-concept predict academic achievement: Evidence from a selective student population. Learning and Individual Differences, 19, 596-608. DOI: https://doi.org/10.1016/j.lindif.2009.08.001

Lamas, H. (2015). Sobre el rendimiento escolar. Propósitos y Representaciones, 3(1), 313-386. DOI: https://doi.org/10.20511/pyr2015.v3n1.74

Llor, L., Ferrando, M., Ferrándiz, C., Hernández, D., Sáinz, M., Prieto, M. D. y Fernández, M. C. (2012). Inteligencias múltiples y alta habilidad. Aula Abierta, 40, 27-38.

Lynn, R. y Mikk, J. (2009). National IQs predict educational attainment in math, reading and science across 56 nations. Intelligence, 37(3), 305-310. DOI: https://doi.org/10.1016/j. intell.2009.01.002

McKenzie, W. (1999). Multiples Intelligences and Instructional Technology. Washington: Iste Publications.

Morales, M. C. (2013). Inteligencias múltiples y rendimiento académico en alumnos de 2 de E.S.O. Propuesta de un programa de mejora (trabajo final de máster). Universidad Internacional de La Rioja, Villafranca de los Barros, España.

Núñez, M. C. y Lozano, I. (2005). Evolución del rendimiento matemático temprano en una muestra de alumnos con discapacidad intelectual mediante la prueba Tema-2. Infancia y Aprendizaje, 28(1), 39-50. DOI: https://doi. org/10.1174/0210370053125533 
Núñez, T., Bryant, P., Evans, D., Bell, D., Gardner, S., Gradner, A. y Carraher, J. (2007). The contribution of logical reasoning to the learning of mathematics in primary school. British Journal of Developmental Psychology, 25(1), 147-166. DOI: https://doi. org/10.1348/026151006X153127

Otero, C., Martín, E., León del Barco, B. y Vicente, F. (2009). Inteligencia emocional y rendimiento académico en estudiantes de enseñanza secundaria. Diferencias de género. Revista Galego-Portuguesa de Psicoloxía e Educación, 17, 275-284

Prieto, M. D. y Ballester, P. (2003). Las inteligencias múltiples. Diferentes formas de enseñar y aprender. Madrid: Pirámide.

Ramos-Amaya, M. D. y Martínez-Berruezo, M. Á. (2015). Análisis de inteligencias múltiples en el alumnado de educación secundaria. Un enfoque neuropsicológico. Recuperado de http:// research.unir.net/wp-content/uploads/2015/07/ Martinez-berruezoRAMOS-AMAYA-000000031.pdf

Rincón, M. M. (2005). Inteligencias múltiples y rendimiento académico en alumnos de educación básica de la U.E. Nuestra Señora de Chiquinquirá. H.H. Maristas (tesis de maestría). Universidad Rafael Urdaneta, Maracaibo, Venezuela.

Stock, P., Desoete, A. y Roeyers, H. (2009). Predicting arithmetic abilities: The role of preparatory arithmetic markers and inteIligence. Journal of Psychoeducational Assessment, 27(3), 237-251. DOI: https://doi. org/10.1177/0734282908330587 